\title{
Transgenic overexpression of transient receptor potential vanilloid subtype 1 attenuates isoproterenol-induced myocardial fibrosis in mice
}

\author{
QIANG WANG ${ }^{*}$, YUNRONG ZHANG* ${ }^{*}$ DE LI, YAN ZHANG, BING TANG, \\ GANG LI, YONGJIAN YANG and DACHUN YANG \\ Department of Cardiology, Chengdu Military General Hospital, Jinniu, Chengdu, Sichuan 610083, P.R. China
}

Received August 8, 2015; Accepted June 8, 2016

DOI: $10.3892 /$ ijmm.2016.2648

\begin{abstract}
Transient receptor potential vanilloid subtype 1 (TRPV1) is a non-selective cation channel with high permeability to $\mathrm{Ca}^{2+}$. Intracellular $\mathrm{Ca}^{2+}$ signaling is an essential regulator of endothelial nitric oxide (NO) synthase (eNOS) that plays a beneficial role in myocardial fibrosis. The aim of the present study was to determine the role of TRPV1 in isoproterenol-induced myocardial fibrosis. Transgenic mice overexpressing TRPV1 were generated on a C57BL/6J genetic background. An animal model of myocardial fibrosis was created by subcutaneously injecting the mice with isoproterenol. We found that the wild-type mice exhibited a significant increase in heart/body weight ratio, left ventricle/body weight ratio, left ventricular end-diastolic pressure (LVEDP), the cardiac fibrotic lesion area and collagen content, as well as a marked decrease in eNOS phosphorylation and $\mathrm{NO} /$ cyclic guanosine monophosphate (cGMP) levels at 2 weeks after the administration of isoproterenol (all $\mathrm{p}<0.01$ ). However, these changes were significantly attenuated in the TRPV1 transgenic mice $(\mathrm{p}<0.05$ or $\mathrm{p}<0.01)$. Moreover, the beneficial effects on myocardial fibrosis exerted by the overexpression of TRPV1 were attenuated by the administration of the eNOS inhibitor, $N \omega$-nitro-L-arginine methyl ester (L-NAME) (all $\mathrm{p}<0.05)$. Similar anti-fibrotic effects were observed in in vitro experiments with primary cultured cardiac fibroblasts. The findings
\end{abstract}

Correspondence to: Dr Dachun Yang or Dr Yongjian Yang, Department of Cardiology, Chengdu Military General Hospital, 270 Tianhui Road, Rongdu Avenue, Jinniu, Chengdu, Sichuan 610083, P.R. China

E-mail: yangdc71@126.com

E-mail: yangyongjian38@yahoo.com

${ }^{*}$ Contributed equally

Key words: endothelial nitric oxide synthase, calcium, ventricular dysfunction, myocardial fibrosis, transient receptor potential vanilloid subtype 1 of our study suggest that TRPV1 overexpression attenuates isoproterenol-induced myocardial fibrosis.

\section{Introduction}

The common pathological pathway of heart failure is myocardial remodeling, including the hypertrophy of cardiomyocytes and hyperplasia of fibroblasts that subsequently causes the accumulation of extracellular matrix components, such as collagen and leads to myocardial fibrosis (1). Severe fibrosis results in increased myocardial stiffness, causing ventricular dysfunction and, ultimately, heart failure (2). No effective treatment for cardiac fibrosis has yet been established (3).

Isoproterenol, a selective $\beta$-adrenoceptor agonist, has been used to induce a mouse model of cardiac fibrosis (4). Adrenergic stimulation induces cardiac fibrosis partly by stimulating fibroblast proliferation (5). Nitric oxide (NO) produced by endothelial NO synthase (eNOS) inhibits adrenergic stimulation (6). Human eNOS gene delivery has been shown to protect against cardiac remodeling following myocardial infarction (7). However, the effect of eNOS activation on isoproterenol-induced myocardial fibrosis is not fully understood.

Intracellular calcium and calmodulin-dependent protein kinase play an important role in the activation of eNOS $(8,9)$. Changes in the concentration of free cytosolic $\mathrm{Ca}^{2+}$ are of fundamental importance in eNOS activation (10). The transient receptor potential (TRP) superfamily consists of a large number of cation channels that are modulators of intracellular $\mathrm{Ca}^{2+}$ signaling (11). TRP vanilloid subtype 1 (TRPV1), a non-selective cation channel with significant permeability to $\mathrm{Ca}^{2+}$, was initially identified as the receptor of capsaicin, with high expression in sensory neurons and heart cells, including cardiac fibroblasts (12). Although TRPV1 has been shown to be involved in the regulation of several types of cardiovascular diseases, including hypertension, atherosclerosis and cardiac arrhythmias (13), the role of TRPV1 in isoproterenol-induced cardiac fibrosis has not been yet elucidated.

Collectively, we hypothesized that TRPV1 may regulate cardiac fibrosis by regulating $\mathrm{Ca}^{2+}$ entry and the eNOS/NO signaling pathway. The present study was designed to determine whether the transgenic overexpression of TRPV1 affects isoproterenol-induced cardiac fibrosis in mice. 


\section{Materials and methods}

Transgenic mice. The handling of animals and all experimental procedures were approved by the Institutional Animal Care and Use Committee of the Chengdu Military General Hospital. Transgenic founder mice were generated on a C57BL/6J background according to our previous study (14). The cDNA of TRPV1 driven by the elongation factor $1 \alpha(\mathrm{EF} 1 \alpha)$ promoter was cloned into the pDONR221 vector (Cyagen Biosciences Inc., Guangzhou, China). The resulting plasmid, pDown-TRPV1, was confirmed by restriction enzyme analysis and DNA sequencing. The linearized pRP.EX3d-TRPV1 was purified from agarose gel using a QIAquick Gel Extraction kit (Qiagen, Chatsworth, CA, USA), adjusted to a final concentration of $1 \mathrm{mg} / \mathrm{ml}$ in Tris-EDTA buffer and used as a DNA solution for microinjection. The fertilized one-cell eggs from hormonally superovulated female C57BL/6J mice (from Cyagen Biosciences Inc.; $n=20$ ) were micro-injected with the DNA solution under a microscope. The injected fertilized eggs were transplanted into the oviducts of pseudo-pregnant mice (foster mothers; C57BL/6J mice), which gave birth to offspring. The offspring mice carrying the TRPV1 cDNA detected by PCR analysis were considered the transgenic founder mice.

Animal care. Male C57BL/6J wild-type mice were purchased from Dashuo Biotech Inc., Chengdu, China. The transgenic mice were mated with the C57BL/6J wild-type mice, and their offspring were genotyped to obtain the transgenic and wild-type mice for the experiments. Male C57BL/6J wildtype and TRPV1 transgenic mice 6-8 weeks of age were housed under a 12/12-h day/night cycle, with access to food and water ad libitum. The mice were randomly assigned to the following groups: i) the control group $(n=16)$ : mice received a daily injection of $0.9 \%$ saline subcutaneously; ii) the isoproterenol group $(\mathrm{n}=16)$ : mice received $3 \mathrm{mg} / \mathrm{kg}$ isoproterenol subcutaneously once daily, as previously described (15); and iii) the isoproterenol plus $N \omega$-nitro-L-arginine methyl ester (L-NAME) (Sigma-Aldrich, St. Louis, MO, USA) group $(\mathrm{n}=16)$ : mice received L-NAME $(1 \mathrm{mg} / \mathrm{ml})$ in their drinking water, as previously described (16). At 2 weeks after the treatment, the mice were sacrificed by cervical dislocation under deep anesthesia with pentobarbital $(60 \mathrm{mg} / \mathrm{kg})$ and the hearts of 8 mice from each group were harvested and weighed to calculate the heart/body weight ratio. The atrial tissue and right ventricle were then removed from the hearts to obtain the left ventricle for calculating the left ventricular/body weight ratio and for further measurements. Additionally, the hearts from the other 8 mice were used for histological examination.

Measurement of blood pressure (BP). Indirect systolic and diastolic BP and heart rate measurements were performed in conscious, restrained mice by tail-cuff plethysmography (BP-2010A blood pressure system; Softron Biotechnology, Beijing, China).

Hemodynamics. The mice were anesthetized with urethane $(1.2 \mathrm{~g} / \mathrm{kg}$, intraperitoneally). A microconductance pressure catheter (ARIA SPR-853; Millar Instruments, Inc., Houston, TX, USA) was introduced through the right carotid artery into the left ventricle as previously described (15). Data were collected on Chart via PowerLab (AD Instruments Pty, Ltd., Castle Hill, Australia). The left ventricular end-systolic pressure (LVESP), left ventricular end-diastolic pressure (LVEDP) and left ventricular $\mathrm{dP} / \mathrm{dt}_{\max / \min }$ were calculated.

Histological analysis. The hearts were excised and retrograde-perfused with phosphate-buffered saline and embedded in paraffin. The paraffin-embedded hearts were cut into 5- $\mu \mathrm{m}$-thick sections which were stained with Masson's trichrome. The blue-stained area presents fibrosis (15).

Culture of cardiac fibroblasts. Primary adult mouse cardiac fibroblasts were isolated by proteolytic dissociation of the ventricular tissue of the transgenic mice and the wild-type littermates and cultured using standard protocols as previously described (15). Cardiac fibroblasts were harvested and cultured in Dulbecco's modified Eagle's medium (DMEM) supplemented with $10 \% \mathrm{FBS}, 2 \mathrm{mM}$ L-glutamine, $100 \mu \mathrm{g} / \mathrm{ml}$ penicillin $\mathrm{G}, 100 \mu \mathrm{g} / \mathrm{ml}$ streptomycin and $100 \mathrm{U} / \mathrm{ml}$ penicillin. Cardiac fibroblasts during early passages were used. One day before the experiments, the cells were serum-starved. TRPV1 expression in the cultured cardiac fibroblast was detected by immunohistochemical staining with primary anti-TRPV1 antibody (1:200 dilution) (ab111973; Abcam, Cambridge, UK) and stained using a DAB detection kit. The cardiac fibroblasts were incubated with the vehicle (saline), $10 \mu \mathrm{M}$ isoproterenol or isoproterenol plus $100 \mu \mathrm{M} \mathrm{L}-\mathrm{NAME}$ for $24 \mathrm{~h}$.

Measurement of cytosolic calcium. The cytosolic free calcium concentration was measured in the cultured fibroblasts as previously described (17). Briefly, the cells were loaded with $2 \mu \mathrm{M}$ of fura2/AM (Sigma-Aldrich) at room temperature for $60 \mathrm{~min}$ and washed to remove the extraneous dye. Fluorescence was recorded at $510 \mathrm{~nm}$ emission with excitation wavelengths of 340 and $380 \mathrm{~nm}$ in baseline and following stimulation with capsaicin $(100 \mathrm{nM})$ and the fluorescence excitation ratio was then calculated.

Western blot analysis. Protein lysates were obtained by homogenizing myocardial tissues or cardiac fibroblasts with lysis buffer according to our previous study (18). The protein concentration was determined with Bio-Rad protein assay reagent (Bio-Rad Laboratories, Inc., Hercules, CA, USA). Equal amounts of protein extracts were separated by SDS-polyacrylamide gels (8-12\%). The samples were then transferred onto PVDF membranes (Millipore Corp., Billerica, MA, USA). The membranes were blocked with TBS-T containing 5\% skim powdered milk for $1 \mathrm{~h}$ and then incubated with anti-TRPV1 (1:500 dilution; ab74855) (Abcam), anti-collagen I (1:250 dilution; sc-8784), anticollagen III (1:300 dilution; sc-8781), anti-fibronectin (1:300 dilution; sc-9068) (all from Santa Cruz Biotechnology, Inc., Santa Cruz, CA, USA), anti-phospho-Akt (1:800 dilution; 9271), anti-Akt (1:700 dilution; 9272), anti-phospho-eNOS (1:1,000 dilution; 9571), anti-eNOS (1:1,000 dilution; 9572) (all from Cell Signaling Technology, Inc., Danvers, MA, USA), and anti-GAPDH (1:200; BA2913) (Boster, Wuhan, China) antibodies overnight at $4^{\circ} \mathrm{C}$. The membranes were incubated with a horseradish peroxidase-conjugated secondary antibody (1:1,000 dilution) (Santa Cruz Biotechnology, Inc.). The membranes were then analyzed using an ECL chemiluminescence kit 
(Amersham Biosciences, Uppsala, Sweden). The results of western blot analysis were quantified using NIH Image software version 1.61. Values are expressed as relative value units.

Real-time polymerase chain reaction (RT-PCR). RT-PCR was performed using the One Step SYBR PrimeScript RT-PCR kit II (RR086A) (Takara Bio, Inc., Otsu, Japan). The relative amount of mRNA was calculated by $2^{-\Delta \Delta C T}$ and was normalized to the housekeeping gene, GAPDH. Each sample was run and analyzed in triplicate. The sequences of the primers used for PCR were as follows: mouse collagen I forward, 5'-gcg agt gct gtg ctt tct g-3' and reverse, 5'-tcc ctc gac tcc tac atc ttc-3'; mouse collagen III forward, 5 '-ccc aac cca gag atc cca tt-3' and reverse, 5'-gaa gca cag gag cag gtg tag a-3'; mouse fibronectin forward, $5^{\prime}$-tct ggg aaa tgg aaa agg gga atg g-3' and reverse, 5'-cac tga agc agg ttt cct cgg tgt-3'; and mouse GAPDH forward, 5'-gac atc aag aag gtg gtg aag c-3' and reverse, 5'-gaa ggt gga aga gtg gga gtt-3'.

Measurements of cardiac NO and cyclic guanosine monophosphate (cGMP) levels. The frozen left ventricles excised from the mice and the cardiac fibroblasts were homogenized in lysis buffer for the measurements of NO and cGMP levels. The NO levels were determined using the Total Nitrate/Nitrite Fluorometric assay kit, and the cGMP levels were quantified using the acetylation protocol for a competitive cGMP enzyme immunoassay (both from Cayman Chemical Co., Ann Arbor, MI, USA).

Cell proliferation assay. Cell proliferation was measured using a CCK-8 cell proliferation kit (Dojindo Laboratories, Kumamoto, Japan) as previously described (19). The cells were seeded onto a 96-well plate with $100 \mu \mathrm{l}$ complete medium and cultured at $37^{\circ} \mathrm{C}$. CCK-8 solution $(10 \mu \mathrm{l})$ was added to each well. The plates were incubated at $37^{\circ} \mathrm{C}$ for $2 \mathrm{~h}$, and the absorbance at $450 \mathrm{~nm}$ was then measured using a microplate reader (Multiskan MK3-Thermo labsystems; Thermo Fisher Scientific, Waltham, MA, USA).

Statistical analysis. Data are presented as the means \pm SEM. Comparisons between groups were determined by one-way ANOVA with the Student's t-test post hoc test (SPSS Inc., Chicago, IL, USA). Results were considered significant when the P-value was $<0.05$.

\section{Results}

Establishment of transgenic mice. Human TRPV1 cDNA was micro-injected into the male pronuclei of fertilized oocytes of C57BL/6J mice. The injected eggs were implanted into the oviducts of pseudo-pregnant foster mothers, which gave birth to offspring. The offspring mice carrying the TRPV1 cDNA detected by PCR analysis were considered the transgenic founder mice (Fig. 1A). Western blot analysis confirmed that TRPV1 protein was significantly overexpressed in the hearts of the transgenic mice compared with those of the wild-type mice ( $\mathrm{p}<0.01$; Fig. 1B). Additionally, calcium entry induced by capsaicin was also enhanced in the cardiac fibroblasts from the TRPV1 transgenic mice compared with those from the wildtype mice (Fig. 1C).

Effect of TRPVI overexpression on left ventricular function. At 2 weeks, there were no significant differences observed in heart
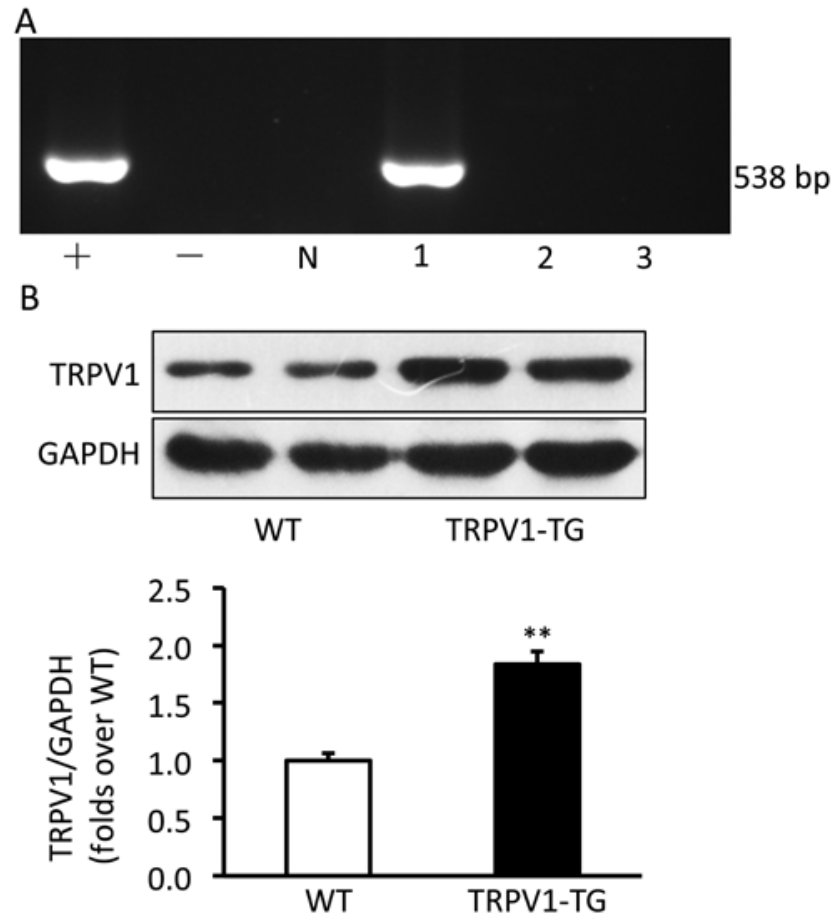

C

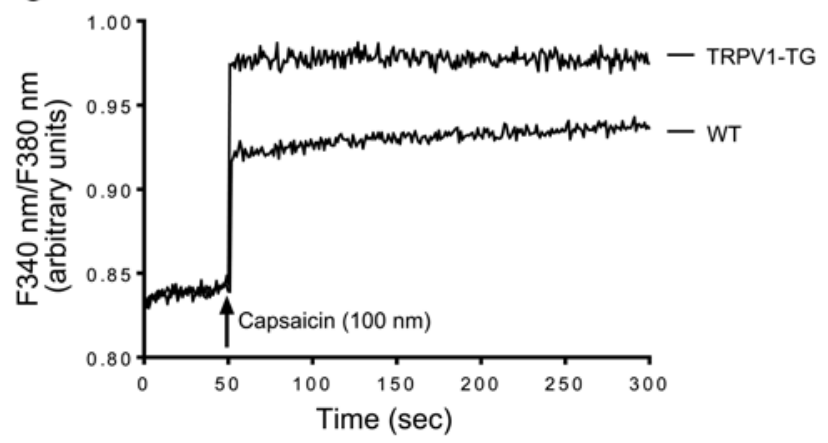

Figure 1. Establishment of transgenic mice. (A) Genotyping of the mice. +, positive control (DNA from transgenic founder mouse); -, negative control (without DNA template); N, wild-type (WT) mice; 1, transgenic mouse; 2 and 3, nontransgenic mice. (B) Western blot analysis of transient receptor potential vanilloid subtype 1 (TRPV1) in myocardial tissue from WT mice and TRPV1 transgenic (TG) mice. ${ }^{* *} \mathrm{p}<0.01$ vs. WT group $(\mathrm{n}=8)$. (C) Calcium entry into cardiac fibroblasts from WT and TRPV1 TG mice.

weight and hemodynamics between the wild-type mice and the transgenic mice (Table I). The wild-type mice exhibited a significant increase in heart/body weight ratio and left ventricle/ body weight ratio at 2 weeks following the administration of isoproterenol (both $\mathrm{p}<0.01$; Table I). However, this increase was significantly attenuated in the TRPV1 transgenic mice when compared to the wild-type mice (both $\mathrm{p}<0.01$; Table I). As expected, the isoproterenol-treated wild-type mice exhibited a significant increase in LVEDP $(\mathrm{p}<0.01)$ and a marked decrease in $\mathrm{dP} / \mathrm{dt}_{\min }(\mathrm{p}<0.01)$; these effects were blunted in the TRPV1 transgenic mice (Table I). However, isoproterenol failed to affect LVESP and $\mathrm{dP} / \mathrm{dt}_{\max }$ in both types of mice (Table I).

Effect of TRPV1 overexpression on cardiac fibrosis. Masson trichrome staining revealed severe interstitial fibrosis within the myocardium of the isoproterenol-treated wild-type mice, while isoproterenol-induced myocardial fibrosis was obviously attenuated in the TRPV1 transgenic mice (Fig. 2A and B). 
A

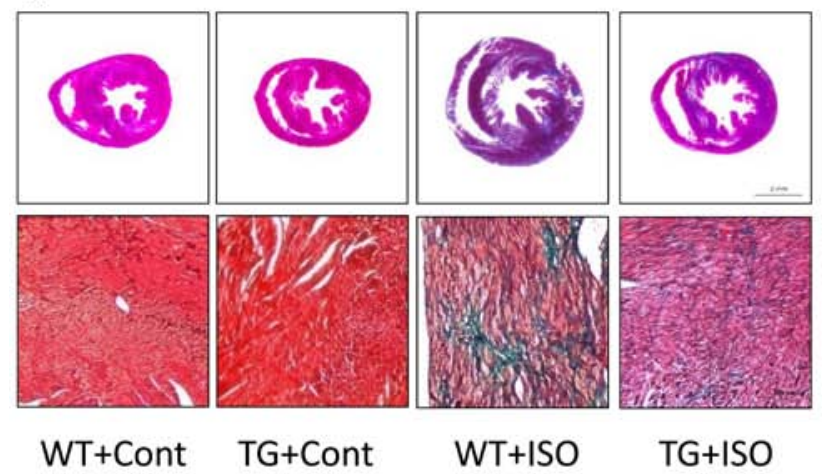

B

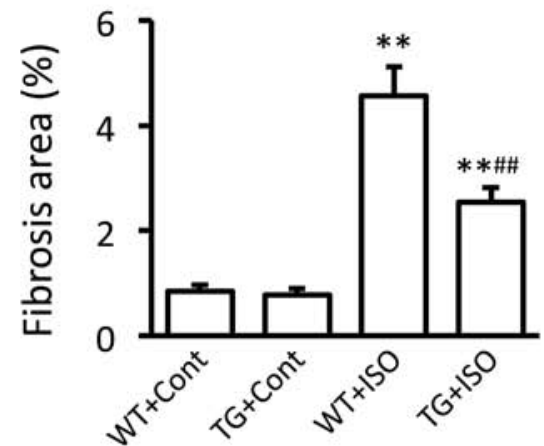

C
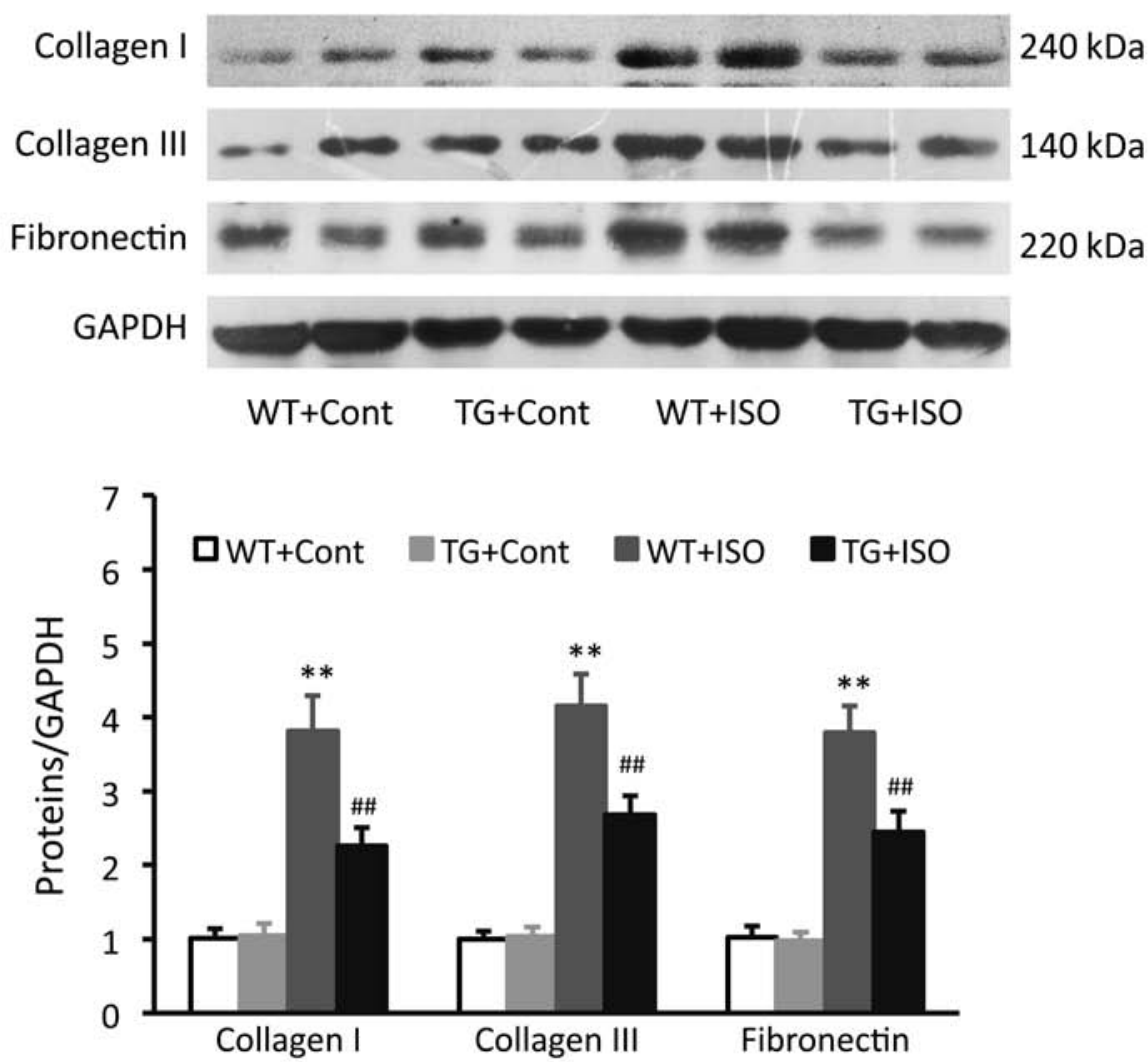

Figure 2. Effect of transient receptor potential vanilloid subtype 1 (TRPV1) on cardiac fibrosis. (A) Representative Masson's trichrome staining of the ventricular tissue from wild-type (WT) and TRPV1 transgenic (TG) mice in control (Cont) or isoproterenol (ISO) group. (B) Quantification of fibrosis. (C) Representative western blots of collagen I and III, and fibronectin in myocardial tissue from mice in the 4 groups. Equal protein loading was confirmed using GAPDH antibody. Target proteins/GAPDH ratios are shown in the bar graph. ${ }^{* *} \mathrm{p}<0.01 \mathrm{vs}$. WT-Cont group, ${ }^{\#} \mathrm{p}<0.01 \mathrm{vs}$. WT-ISO group (n=8).

Isoproterenol induced a significant increase in the protein expression levels of collagen I and III, as well as in the expression of fibronectin in the heart tissues from the wildtype mice (all p<0.01; Fig. 2C). However, this increase was significantly prevented in the TRPV1 transgenic mice (all p<0.01; Fig. 2C).

Effect of TRPVI overexpression on the eNOS signaling pathway. In the isoproterenol-treated wild-type mice, significantly decreased expression levels of $\mathrm{p}-\mathrm{Akt}(\mathrm{p}<0.01)$, $\mathrm{p}$-eNOS $(\mathrm{p}<0.01)$ and total eNOS $(\mathrm{p}<0.05)$ were noted in the ventricular tissues (Fig. 3A and B). However, the levels of p-Akt and p-eNOS were significantly increased in the TRPV1 transgenic mice compared with the wild-type mice following the administration of isoproterenol (Fig. 3B). Similar results were obtained in the measurements of the myocardial $\mathrm{NO}$ and cGMP levels (Fig. 3C and D).

Influence of eNOS on the beneficial effects of TRPVI overexpression. In order to confirm the role of eNOS in the beneficial effects induced by TRPV1 overexpression, the eNOS inhibitor, L-NAME, was administered to the mice receiving isoproterenol. We found that the administration of L-NAME for 2 weeks significantly attenuated the TRPV1 overexpressioninduced decrease in LVEDP (Table I) and the increase in $\mathrm{dP} /$ $\mathrm{dt}_{\text {min }}$ (Table I). Moreover, L-NAME also attenuated the TRPV1 overexpression-induced suppression of fibrosis (Fig. 4A and B).

Analysis of cardiac fibroblasts. The expression of TRPV1 in the cultured cardiac fibroblasts was confirmed by 
Table I. Heart weight and hemodynamics of the mice at 2 weeks.

\begin{tabular}{|c|c|c|c|c|c|c|}
\hline Parameter & WT-Cont & TG-Cont & WT-ISO & TG-ISO & WT-ISO-L-NAME & TG-ISO-L-NAME \\
\hline HW/BW (mg/g) & $4.96 \pm 0.08$ & $4.98 \pm 0.04$ & $6.59 \pm 0.23^{\mathrm{a}}$ & $5.67 \pm 0.21^{\mathrm{b}}$ & $6.65 \pm 0.19$ & $6.29 \pm 0.20^{\mathrm{c}}$ \\
\hline LVW/BW (mg/g) & $3.47 \pm 0.07$ & $3.44 \pm 0.06$ & $4.93 \pm 0.23^{\mathrm{a}}$ & $4.12 \pm 0.18^{b}$ & $4.97 \pm 0.18$ & $4.69 \pm 0.19^{c}$ \\
\hline HR (bpm) & $449 \pm 12$ & $451 \pm 14$ & $552 \pm 13^{a}$ & $546 \pm 14$ & $560 \pm 16$ & $557 \pm 17$ \\
\hline $\mathrm{SBP}(\mathrm{mmHg})$ & $105 \pm 4$ & $104 \pm 5$ & $111 \pm 4$ & $108 \pm 5$ & $115 \pm 6$ & $115 \pm 7$ \\
\hline $\mathrm{DBP}(\mathrm{mmHg})$ & $71 \pm 2$ & $69 \pm 3$ & $74 \pm 2$ & $72 \pm 4$ & $75 \pm 4$ & $75 \pm 4$ \\
\hline MAP (mmHg) & $82 \pm 3$ & $81 \pm 3$ & $86 \pm 3$ & $84 \pm 4$ & $89 \pm 4$ & $88 \pm 5$ \\
\hline LVESP (mmHg) & $107.6 \pm 4.4$ & $106.5 \pm 5.0$ & $112.2 \pm 4.8$ & $116.8 \pm 7.3$ & $117.1 \pm 7.9$ & $119.6 \pm 7.4$ \\
\hline LVEDP (mmHg) & $2.8 \pm 0.4$ & $2.7 \pm 0.3$ & $9.4 \pm 0.9^{a}$ & $5.6 \pm 0.6^{\mathrm{b}}$ & $9.9 \pm 1.2$ & $8.1 \pm 1.2^{c}$ \\
\hline $\mathrm{dP} / \mathrm{dt}_{\max }(\mathrm{mmHg} / \mathrm{sec})$ & $11889 \pm 685$ & $12174 \pm 745$ & $12834 \pm 1030$ & $12335 \pm 751$ & $12740 \pm 950$ & $12188 \pm 635$ \\
\hline $\mathrm{dP} / \mathrm{dt}_{\min }(\mathrm{mmHg} / \mathrm{sec})$ & $-11301 \pm 645$ & $-11551 \pm 731$ & $-7416 \pm 359^{a}$ & $-9547 \pm 480^{\mathrm{b}}$ & $-7259 \pm 335$ & $-7933 \pm 398^{c}$ \\
\hline
\end{tabular}

Values are the means \pm SEM $(n=8)$. HW, heart weight; BW, body weight; LVW, left ventricle weight; HR, heart rate; SBP, systolic blood pressure; DBP, diastolic blood pressure; MAP, mean artery pressure; LVESP, left ventricular end-systolic pressure; LVEDP, left ventricular end-diastolic pressure; $\mathrm{dP} / \mathrm{dt}_{\max }$, maximal rate of pressure development; $\mathrm{dP} / \mathrm{dt}_{\min }$, maximal rate of pressure decay; WT, wild-type mice; TG, TRPV1 transgenic mice; Cont, control; ISO, isoproterenol. ${ }^{\mathrm{a} P}<0.01$ vs. WT-Cont; ${ }^{\mathrm{b}} \mathrm{P}<0.01$ vs. WT-ISO; ${ }^{\mathrm{P}} \mathrm{P}<0.05$ vs. TG-ISO.

A

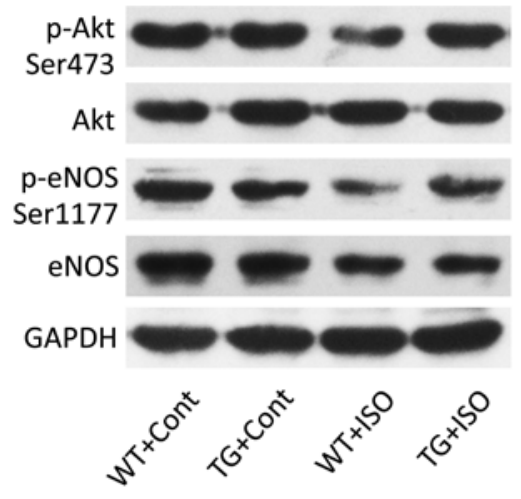

C

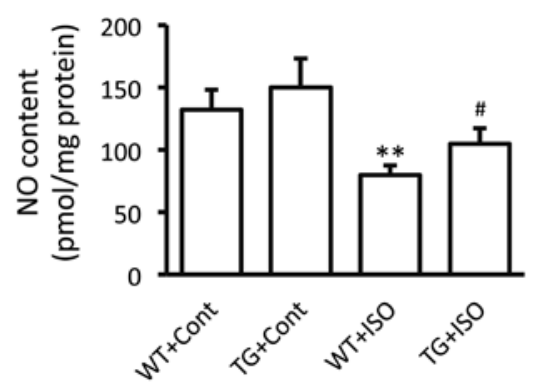

B

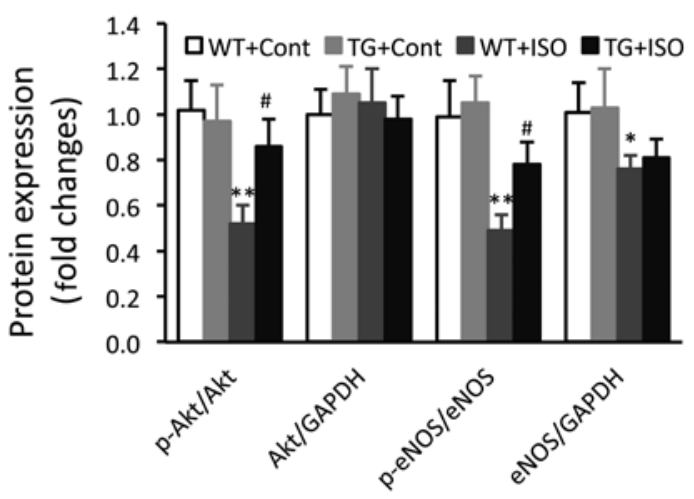

D

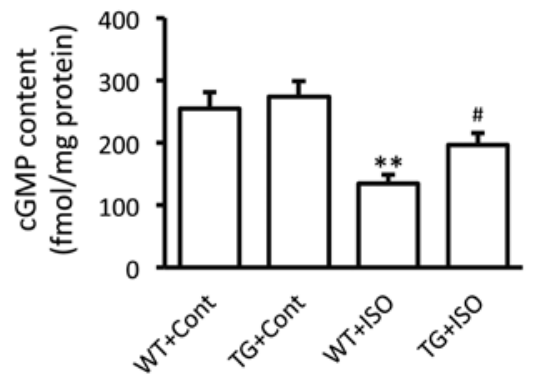

Figure 3. Effect of transient receptor potential vanilloid subtype 1 (TRPV1) on the endothelial nitric oxide synthase (eNOS) signal pathway. (A) Representative western blots of Akt, p-Akt, eNOS and p-eNOS in myocardial tissue from mice in the 4 groups. Equal protein loading was confirmed using GAPDH antibody. (B) Target proteins/GAPDH ratios are shown in the bar graph. (C) NO content and (D) cGMP level in myocardial tissues from the 4 groups were detected. ${ }^{*} \mathrm{p}<0.05,{ }^{* *} \mathrm{p}<0.01 \mathrm{vs}$. WT-Cont group, ${ }^{\#} \mathrm{p}<0.05$ vs. WT-ISO group $(\mathrm{n}=8)$.

immunohistochemical staining (Fig. 5A). Moreover, TRPV1 expression was markedly increased in the cardiac fibroblasts from the transgenic mice compared with those from the wildtype mice (Fig. 5B). Treatment with isoproterenol induced a significant increase in cell proliferation and in the expression of collagen I and III, and fibronectin in the cardiac fibroblasts from the wild-type mice, and these effects were markedly attenuated in the cardiac fibroblasts from the transgenic mice (Fig. 5C-F). Additionally, treatment with L-NAME attenuated the TRPV1 overexpression-induced suppression of fibroblast proliferation and the expression of collagen I and III and fibronectin (Fig. 5C-F). Isoproterenol induced a significant 
A

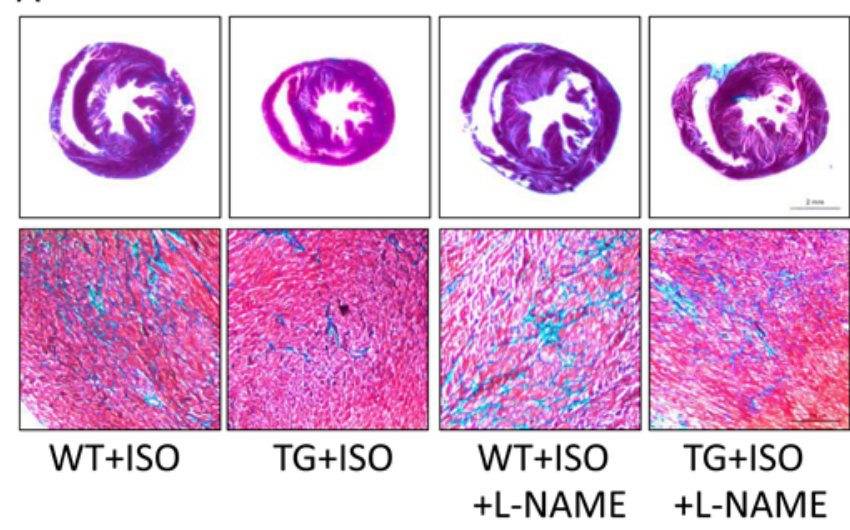

B

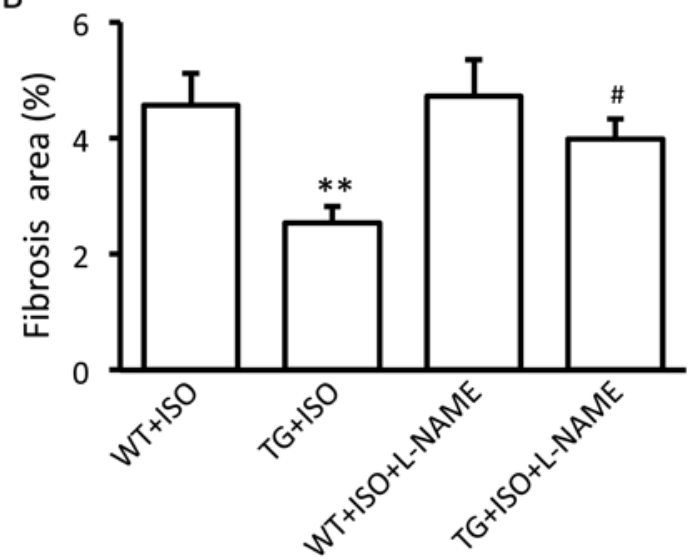

Figure 4. Effect of endothelial nitric oxide synthase (eNOS) on the action of transient receptor potential vanilloid subtype 1 (TRPV1). (A) Representative Masson's trichrome staining of the ventricular tissue and the (B) quantification of fibrosis from wild-type (WT) and TRPV1 transgenic (TG) mice in isoproterenol (ISO) group or ISO plus L-NAME group. ${ }^{* *} \mathrm{p}<0.01$ vs. WT-ISO group, ${ }^{*} \mathrm{p}<0.05$ vs. TG-ISO group ( $\left.\mathrm{n}=8\right)$.

A

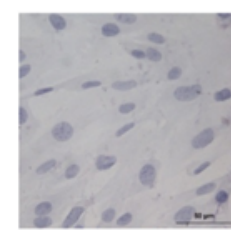

Control

C

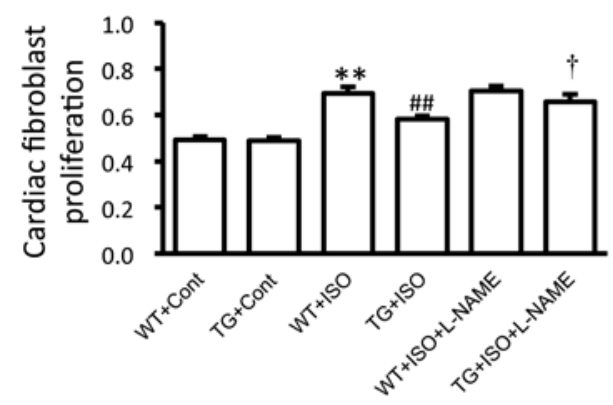

E

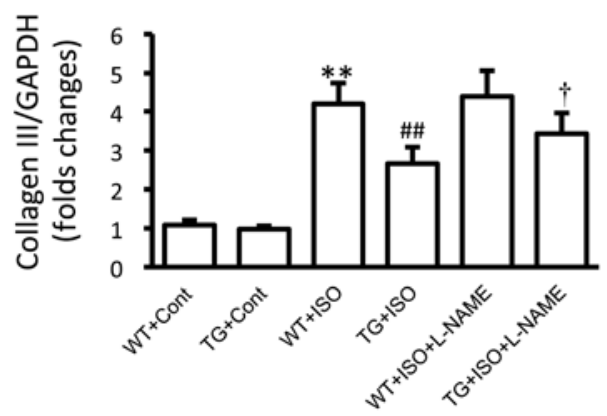

B

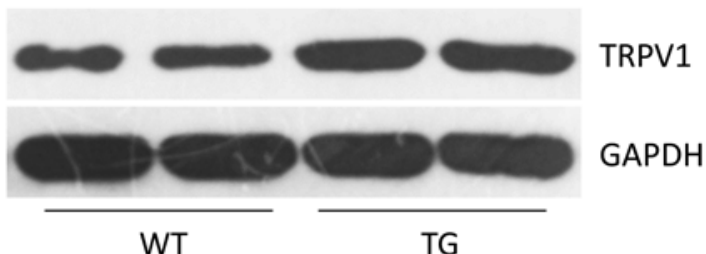

D

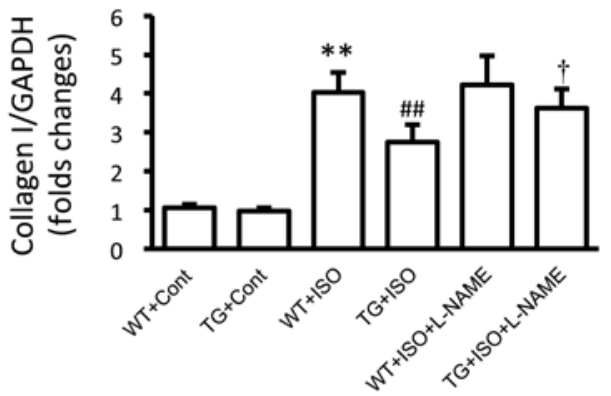

$\mathrm{F}$

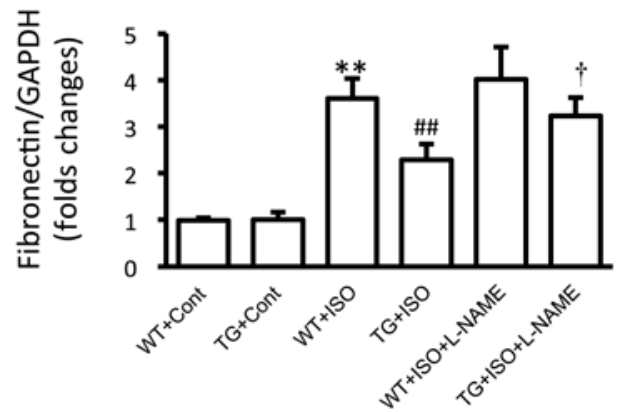

Figure 5. Effects of transient receptor potential vanilloid subtype 1 (TRPV1) on cardiac fibroblast proliferation and collagen production. (A) Immunohistochemical staining of TRPV1 in cardiac fibroblasts. Control, without primary antibody. (B) Representative western blot of TRPV1 in cardiac fibroblasts from wildtype (WT) mice and TRPV1 transgenic (TG) mice. (C) Cell proliferation and mRNA expression of collagen (D) I and (E) III, and (F) fibronectin in cardiac fibroblasts from WT and TG mice treated with the vehicle (Cont), isoproterenol (ISO), or ISO + L-NAME. ${ }^{* *}$ p $<0.01$ vs. WT-Cont group, ${ }^{\# \#<0.01 ~ v s . ~ W T-I S O ~}$ group, ${ }^{\dagger} \mathrm{p}<0.05$ vs. TG-ISO group ( $\mathrm{n}=3$ independent experiments).

downregulation in the levels of p-Akt and p-eNOS and a decrease in the levels of NO and cGMP in the cardiac fibroblasts from wild-type mice, and these effects were partially reversed in the cells from the transgenic mice (Fig. 6A-D). Similarly, L-NAME abolished the TRPV1 overexpression-caused increase in the NO and cGMP levels (Fig. 6C and D). 
A

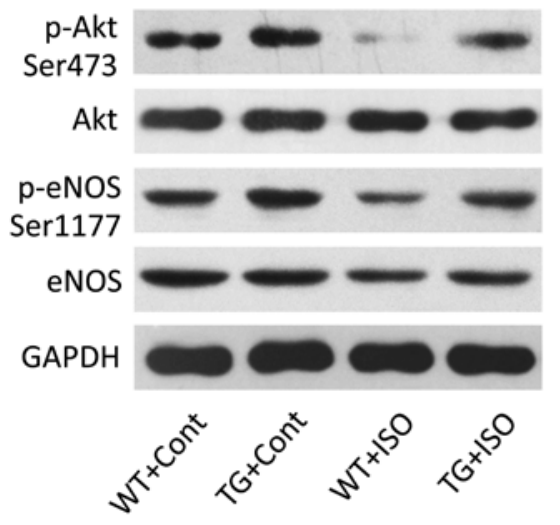

C

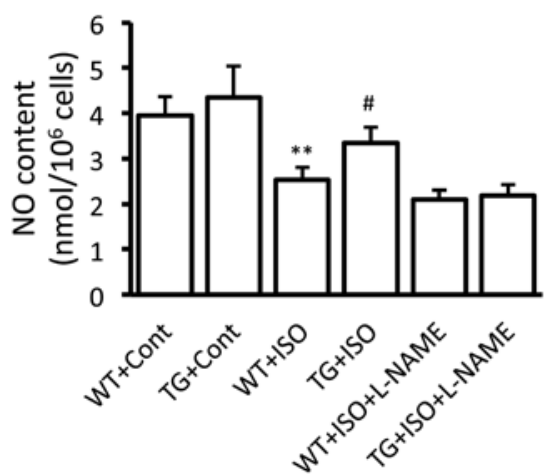

B

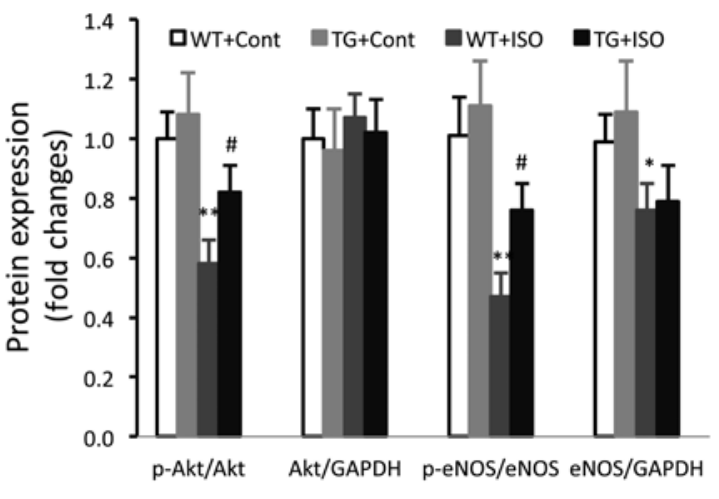

D

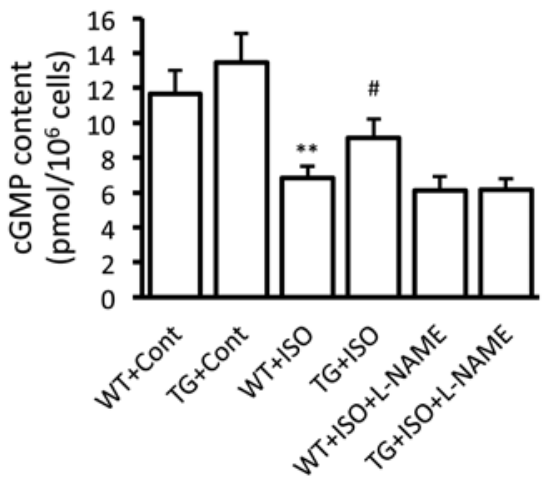

Figure 6. The Akt/endothelial nitric oxide synthase (eNOS)/NO/cGMP pathway in cardiac fibroblasts. (A) Representative western blots of Akt, p-Akt, eNOS and p-eNOS in cardiac fibroblasts from wild-type (WT) mice and transient receptor potential vanilloid subtype 1 (TRPV1) transgenic (TG) mice treated with vehicle (Cont) or isoproterenol (ISO). (B) Target proteins/GAPDH ratios are shown in the bar graph. (C) NO content and (D) cGMP level in cardiac fibroblasts treated with vehicle (Cont), isoproterenol (ISO), or ISO + L-NAME. ${ }^{*} \mathrm{p}<0.05,{ }^{* *} \mathrm{p}<0.01$ vs. WT-Cont group, ${ }^{*} \mathrm{p}<0.05$ vs. WT-ISO group (n=3 independent experiments).

\section{Discussion}

There are three novel findings in the present study: firstly, the transgenic overexpression of TRPV1 attenuated the development of cardiac fibrosis induced by isoproterenol. Secondly, the overexpression of TRPV1 attenuated the isoproterenol-induced impairment of the eNOS/NO/cGMP pathway in myocardial tissue and cardiac fibroblasts. Thirdly, the beneficial effects of TRPV1 on fibrosis and collagen deposition were blunted by the administration of the eNOS inhibitor, L-NAME. These results suggest that TRPV1 overexpression may be able to ameliorate isoproterenolinduce myocardial fibrosis partly through the activation of the eNOS/NO pathway in cardiac fibroblasts.

TRPV1 has been implicated in several types of cardiovascular diseases and metabolic disorders, including hypertension (20), atherosclerosis (21), vascular dysfunction (22), diabetes (23) and obesity (24). The beneficial effects of TRPV1 on ischemia reperfusion injury and on myocardial remodeling and dysfunction have been previously reported by our group and others (19,25-29). Moreover, TRPV1 has also been shown involved in low ambient temperature-induced cardiac hypertrophy and contractile dysfunction and high-salt diet-induced cardiac hypertrophy and fibrosis $(30,31)$. However, there are also a few studies that have demonstrated that TRPV1 may have harmful effects on cardiac hypertrophy and fibrosis using TRPV1-deficient mice $(32,33)$. Therefore, the role of TRPV1 in cardiac hypertrophy and fibrosis has yet to be determined. The present study demonstrated that the overexpression of TRPV1 attenuates isoproterenol-induced cardiac fibrosis. TRPV1 is expressed in several types of cells in the cardiovascular system, including cardiomyocytes, cardiac fibroblasts and endothelial cells $(17,30,34)$. We confirmed the expression of TRPV1 in cardiac fibroblasts and found that the overexpression of TRPV1 exerted anti-fibrotic effects in cell culture experiments. However, we cannot confirm the anti-fibrotic effects induced by TRPV1 overexpression in other types of cells or other organs, since we used global transgenic mice. Moreover, we also cannot rule out the effects of other calcium channels which may be regulated by human TRPV1 overexpression.

The eNOS/NO system provides an ubiquitous protection of the cardiovascular system. It has been reported that isoproterenol reduces the expression and phosphorylation of eNOS (35). The activation of the eNOS/NO pathway plays a beneficial role in either angiotensin II- or isoproterenolinduced cardiac disorders (36-39). Previous studies have demonstrated that $\mathrm{Ca}^{2+}$ signal is essential for the phosphorylation and activation of eNOS. TRP channels, as $\mathrm{Ca}^{2+}$-permeable channels, play a beneficial role in cardiovascular diseases. Moreover, the TRPV1 channel has been included in the activation of eNOS in several previous studies (40-43). The present study demonstrated that TRPV1 overexpression increased the 
phosphorylation of eNOS in the myocardium from isoproterenol-treated mice. We also showed that L-NAME suppressed the beneficial effects of TRPV1 overexpression on fibrosis. A previous study demonstrated that L-NAME enhanced cardiac fibrosis (44). However, L-NAME failed to further increase the fibrosis in isoproterenol-treated mice, suggesting that both L-NAME and isoproterenol may cause fibrosis through the inhibition of eNOS activity.

Collectively, the present study demonstrates that the overexpression of TRPV1 ameliorates isoproterenol-induced cardiac fibrosis and that fibroblasts may play a role in this anti-fibrotic effect.

\section{Acknowledgements}

The present study was supported by grants from the National Natural Science Foundation of China (no. 81170081 awarded to D.Y.), the Military Medical Science Youth Development Project (no. 13QNP058 awarded to Q.W.), the Sichuan Scientific and Technical Innovation Development Project (no. 2015016 awarded to Q.W.), the Chengdu Military Health Academic Leader Foundation (awarded to D.Y.), and the Foundation for Talents of Scientific Research from Chengdu Military General Hospital (awarded to D.Y.).

\section{References}

1. Cohn JN, Ferrari R and Sharpe N: Cardiac remodeling - concepts and clinical implications: a consensus paper from an international forum on cardiac remodeling. Behalf of an International Forum on Cardiac Remodeling. J Am Coll Cardiol 35: 569-582, 2000.

2. Biernacka A and Frangogiannis NG: aging and cardiac fibrosis. Aging Dis 2: 158-173, 2011.

3. Leask A: Potential therapeutic targets for cardiac fibrosis: TGFbeta, angiotensin, endothelin, CCN2, and PDGF, partners in fibroblast activation. Circ Res 106: 1675-1680, 2010.

4. Osadchii OE: Cardiac hypertrophy induced by sustained beta-adrenoreceptor activation: pathophysiological aspects. Heart Fail Rev 12: 66-86, 2007.

5. Nichtova Z, Novotova M, Kralova $E$ and Stankovicova T: Morphological and functional characteristics of models of experimental myocardial injury induced by isoproterenol. Gen Physiol Biophys 31: 141-151, 2012.

6. Ozaki M,Kawashima S, Yamashita T, Hirase T, Ohashi Y,Inoue N, Hirata K and Yokoyama M: Overexpression of endothelial nitric oxide synthase attenuates cardiac hypertrophy induced by chronic isoproterenol infusion. Circ J 66: 851-856, 2002.

7. Smith RS Jr, Agata J, Xia CF, Chao L and Chao J: Human endothelial nitric oxide synthase gene delivery protects against cardiac remodeling and reduces oxidative stress after myocardial infarction. Life Sci 76: 2457-2471, 2005.

8. Park JY, Shin HK, Choi YW, Lee YJ, Bae SS, Han J and Kim CD: Gomisin A induces $\mathrm{Ca}^{2+}$-dependent activation of eNOS in human coronary artery endothelial cells. J Ethnopharmacol 125: 291-296, 2009.

9. Park JH,Lee S, Cho DH, Park YM, Kang DH and Jo I: Far-infrared radiation acutely increases nitric oxide production by increasing $\mathrm{Ca}(2+)$ mobilization and $\mathrm{Ca}(2+) /$ calmodulin-dependent protein kinase II-mediated phosphorylation of endothelial nitric oxide synthase at serine 1179. Biochem Biophys Res Commun 436: 601-606, 2013

10. Gail MH, Boone CW and Thompson CS: A calcium requirement for fibroblast motility and prolifertion. Exp Cell Res 79: 386-390, 1973.

11. Gees M, Colsoul B and Nilius B: The role of transient receptor potential cation channels in $\mathrm{Ca}^{2+}$ signaling. Cold Spring Harb Perspect Biol 2: a003962, 2010.

12. Thilo F, Liu Y, Schulz N, Gergs U, Neumann J, Loddenkemper C, Gollasch $\mathrm{M}$ and Tepel M: Increased transient receptor potential vanilloid type 1 (TRPV1) channel expression in hypertrophic heart. Biochem Biophys Res Commun 401: 98-103, 2010.
13. Marshall NJ, Liang L, Bodkin J, Dessapt-Baradez C, Nandi M, Collot-Teixeira S, Smillie SJ, Lalgi K, Fernandes ES, Gnudi L, and Brain SD: A role for TRPV1 in influencing the onset of cardiovascular disease in obesity. Hypertension 61: 246-252, 2013.

14. Ma S, Li D, Yang D, Tan Y, Tang B, Jin F, Jiang S, Li X and Yang Y: Establishment of a conditional transgenic mouse model expressing human uncoupling protein 2 in vascular smooth muscle cells. Exp Ther Med 4: 545-547, 2012.

15. Ma S, Yang D, Wang K, Tang B, Li D and Yang Y: Cryptotanshinone attenuates isoprenaline-induced cardiac fibrosis in mice associated with upregulation and activation of matrix metalloproteinase-2. Mol Med Rep 6: 145-150, 2012.

16. Mukai Y, Rikitake Y, Shiojima I, Wolfrum S, Satoh M, Takeshita K, Hiroi Y, Salomone S, Kim HH, Benjamin LE, et al: Decreased vascular lesion formation in mice with inducible endothelial-specific expression of protein kinase Akt. J Clin Invest 116: 334-343, 2006.

17. Yang D, Luo Z, Ma S, Wong WT, Ma L, Zhong J, He H, Zhao Z, Cao T, Yan Z, et al: Activation of TRPV1 by dietary capsaicin improves endothelium-dependent vasorelaxation and prevents hypertension. Cell Metab 12: 130-141, 2010.

18. Ma S, Yang D, Li D, Tang B, Sun M and Yang Y: Cardiac extracellular matrix tenascin-C deposition during fibronectin degradation. Biochem Biophys Res Commun 409: 321-327, 2011.

19. Wang Q, Ma S, Li D, Zhang Y, Tang B, Qiu C, Yang Y and Yang D: Dietary capsaicin ameliorates pressure overload-induced cardiac hypertrophy and fibrosis through the transient receptor potential vanilloid type 1. Am J Hypertens 27: 1521-1529, 2014.

20. Andresen MC and Peters JH: TRPV1, hypertension, and cardiovascular regulation. Cell Metab 12: 421, author reply 422, 2010.

21. Zhao JF, Ching LC, Kou YR, Lin SJ, Wei J, Shyue SK and Lee TS: Activation of TRPV1 prevents OxLDL-induced lipid accumulation and TNF- $\alpha$-induced inflammation in macrophages: role of liver X receptor $\alpha$. Mediators Inflamm 2013: 925171, 2013

22. Ohanyan VA, Guarini G, Thodeti CK, Talasila PK, Raman P, Haney RM, Meszaros JG, Damron DS and Bratz IN: Endothelin-mediated in vivo pressor responses following TRPV1 activation. Am J Physiol Heart Circ Physiol 301: H1135-H1142, 2011.

23. Suri A and Szallasi A: The emerging role of TRPV1 in diabetes and obesity. Trends Pharmacol Sci 29: 29-36, 2008.

24. Motter AL and Ahern GP: TRPV1-null mice are protected from diet-induced obesity. FEBS Lett 582: 2257-2262, 2008.

25. Wang L and Wang DH: TRPV1 gene knockout impairs postischemic recovery in isolated perfused heart in mice. Circulation 112: 3617-3623, 2005.

26. Zhong B and Wang DH: TRPV1 gene knockout impairs preconditioning protection against myocardial injury in isolated perfused hearts in mice. Am J Physiol Heart Circ Physiol 293: H1791-H1798, 2007.

27. Huang W, Rubinstein J, Prieto AR, Thang LV and Wang DH: Transient receptor potential vanilloid gene deletion exacerbates inflammation and atypical cardiac remodeling after myocardial infarction. Hypertension 53: 243-250, 2009.

28. Huang W, Rubinstein J, Prieto AR and Wang DH: Enhanced postmyocardial infarction fibrosis via stimulation of the transforming growth factor-beta-Smad2 signaling pathway: role of transient receptor potential vanilloid type 1 channels. J Hypertens 28: 367-376, 2010.

29. Ren JY, Song JX, Lu MY and Chen H: Cardioprotection by ischemic postconditioning is lost in isolated perfused heart from diabetic rats: involvement of transient receptor potential vanilloid 1, calcitonin gene-related peptide and substance $P$. Regul Pept 169: 49-57, 2011.

30. Zhang Y, Li L, Hua Y, Nunn JM, Dong F, Yanagisawa M and Ren J: Cardiac-specific knockout of ET(A) receptor mitigates low ambient temperature-induced cardiac hypertrophy and contractile dysfunction. J Mol Cell Biol 4: 97-107, 2012.

31. Gao F, Liang Y, Wang X, Lu Z, Li L, Zhu S, Liu D, Yan Z and Zhu Z: TRPV1 activation attenuates high-salt diet-induced cardiac hypertrophy and fibrosis through PPAR- $\delta$ upregulation. PPAR Res 2014: 491963, 2014.

32. Buckley CL and Stokes AJ: Mice lacking functional TRPV1 are protected from pressure overload cardiac hypertrophy. Channels (Austin) 5: 367-374, 2011.

33. Horton JS, Buckley CL and Stokes AJ: Successful TRPV1 antagonist treatment for cardiac hypertrophy and heart failure in mice. Channels (Austin) 7: 17-22, 2013. 
34. Ge W, Yuan M, Ceylan AF, Wang X and Ren J: Mitochondrial aldehyde dehydrogenase protects against doxorubicin cardiotoxicity through a transient receptor potential channel vanilloid 1-mediated mechanism. Biochim Biophys Acta 1862: 622-634, 2016.

35. Bhuiyan MS, Shioda N and Fukunaga K: Chronic beta-AR activation-induced calpain activation and impaired eNOS-Akt signaling mediates cardiac injury in ovariectomized female rats. Expert Opin Ther Targets 13: 275-286, 2009.

36. Belge C, Hammond J, Dubois-Deruy E, Manoury B, Hamelet J, Beauloye C, Markl A, Pouleur AC, Bertrand L, Esfahani H, et al: Enhanced expression of $\beta 3$-adrenoceptors in cardiac myocytes attenuates neurohormone-induced hypertrophic remodeling through nitric oxide synthase. Circulation 129: 451-462, 2014.

37. Savergnini SQ, Ianzer D, Carvalho MB, Ferreira AJ, Silva GA, Marques FD, Peluso AA, Beiman M, Cojocaru G, Cohen Y, et al: The novel Mas agonist, CGEN-856S, attenuates isoproterenol-induced cardiac remodeling and myocardial infarction injury in rats. PLoS One 8: e57757, 2013.

38. Bharti S, Singh R, Chauhan SS, Hussain T, Al-Attas OS and Arya DS: Phosphorylation of Akt/GSK-3ß/eNOS amplifies 5-HT2B receptor blockade mediated anti-hypertrophic effect in rats. FEBS Lett 586: 180-185, 2012.

39. Liao Y, Asakura M, Takashima S, Ogai A, Asano Y, Shintani Y, Minamino T, Asanuma H, Sanada S, Kim J, et al: Celiprolol a vasodilatory beta-blocker, inhibits pressure overload-induced cardiac hypertrophy and prevents the transition to heart failure via nitric oxide-dependent mechanisms in mice. Circulation 110 692-699, 2004
40. Ching LC, Kou YR, Shyue SK, Su KH, Wei J, Cheng LC, Yu YB, Pan CC and Lee TS: Molecular mechanisms of activation of endothelial nitric oxide synthase mediated by transient receptor potential vanilloid type s1. Cardiovasc Res 91: 492-501, 2011.

41. Ching LC, Chen CY, Su KH, Hou HH, Shyue SK, Kou YR and Lee TS: Implication of AMP-activated protein kinase in transient receptor potential vanilloid type 1-mediated activation of endothelial nitric oxide synthase. Mol Med 18: 805-815, 2012.

42. Su KH, Lin SJ, Wei J, Lee KI, Zhao JF, Shyue SK and Lee TS: The essential role of transient receptor potential vanilloid $\mathrm{s} 1$ in simvastatin-induced activation of endothelial nitric oxide synthase and angiogenesis. Acta Physiol (Oxf) 212: 191-204, 2014.

43. Guarini G, Ohanyan VA, Kmetz JG, DelloStritto DJ, Thoppil RJ, Thodeti CK, Meszaros JG, Damron DS and Bratz IN: Disruption of TRPV1-mediated coupling of coronary blood flow to cardiac metabolism in diabetic mice: role of nitric oxide and BK channels. Am J Physiol Heart Circ Physiol 303: H216-H223, 2012.

44. Kazakov A, Hall R, Jagoda P, Bachelier K, Müller-Best P, Semenov A, Lammert F, Böhm M and Laufs U: Inhibition of endothelial nitric oxide synthase induces and enhances myocardial fibrosis. Cardiovasc Res 100: 211-221, 2013. 